OPEN ACCESS

Edited by:

Changiz Geula,

Northwestern University,

United States

Reviewed by:

Chen Ding,

Yale University, United States

Bertrand Glize,

Université de Bordeaux, France

*Correspondence:

Shuren Li

shuren.li@meduniwien.ac.at

Jingling Chang

ear6979@163.com

Received: 22 November 2020 Accepted: 16 November 2021

Published: 16 December 2021

Citation:

Fan $R$, Gao $Y$, Zhang $H$, Xin $X$,

Sang F, Tan Z, Zhang B, Li X,

Huang X, Li S and Chang J (2021)

Lesion Distribution and Early Changes

of Right Hemisphere in Chinese

Patients With Post-stroke Aphasia.

Front. Aging Neurosci. 13:632217.

doi: 10.3389/fnagi.2021.632217

\section{Lesion Distribution and Early Changes of Right Hemisphere in Chinese Patients With Post-stroke Aphasia}

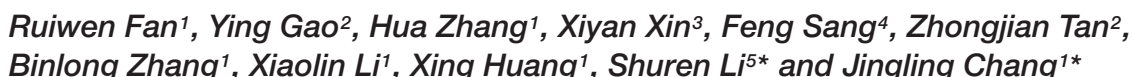

${ }^{1}$ Department of Neurology, Dongzhimen Hospital, Beijing University of Chinese Medicine, Beijing, China, ${ }^{2}$ Key Laboratory of Encephalopathy Treatment of Chinese Medicine, State Administration of Traditional Chinese Medicine of the People's Republic of China, Beijing, China, ${ }^{3}$ TCM Department of Peking University Third Hospital, Peking University, Beijing, China, ${ }^{4}$ State Key Laboratory of Cognitive Neuroscience and Learning, Beijing Normal University, Beijing, China, ${ }^{5}$ Division of Nuclear Medicine, Department of Biomedical Imaging and Image-Guided Therapy, Medical University of Vienna, Vienna, Austria

The role of the right hemisphere $(\mathrm{RH})$ in post-stroke aphasia (PSA) has not been completely understood. In general, the language alterations in PSA are normally evaluated from the perspective of the language processing models developed from Western languages such as English. However, the successful application of the models for assessing Chinese-language functions in patients with PSA has not been reported. In this study, the features of specific language-related lesion distribution and early variations of structure in $\mathrm{RH}$ in Chinese patients with PSA were investigated. Forty-two aphasic patients (female: 13, male: 29, mean age: $58 \pm 12$ years) with left hemisphere $(\mathrm{LH})$ injury between 1 and 6 months after stroke were included. The morphological characteristics, both at the levels of gray matter (GM) and white matter (WM), were quantified by $3 T$ multiparametric brain MRI. The Fridriksson et al.'s dual-stream model was used to compare language-related lesion regions. Voxel-based lesion-symptom mapping (VLSM) analysis has been performed. Our results showed that lesions in the precentral, superior frontal, middle frontal, and postcentral gyri were responsible for both the production and comprehension dysfunction of Chinese patients with PSA and were quite different from the lesions described by using the dual-stream model of Fridriksson et al. Furthermore, gray matter volume (GMV) was found significantly decreased in $\mathrm{RH}$, and WM integrity was disturbed in $\mathrm{RH}$ after LH injury in Chinese patients with PSA. The different lesion patterns between Chinese patients with PSA and English-speaking patients with PSA may indicate that the dual-stream model of Fridriksson et al. is not suitable for the assessment of Chinese-language functions in Chinese patients with PSA in subacute phase of recovery. Moreover, decreased structural integrity in $\mathrm{RH}$ was found in Chinese patients with PSA.

Keywords: aphasia, stroke, language, structural covariation, MRI, right hemisphere 


\section{INTRODUCTION}

Stroke is one of the leading causes of aphasia, accounting for approximately $30-40 \%$ of stroke survivors with aphasia (Engelter et al., 2006; Marebwa et al., 2017; Nouwens et al., 2018). The recovery of language function after stroke may be divided into three overlapping phases. The initial phase is called the acute phase and lasts for about 2 weeks after the onset of the stroke. The second phase is called the subacute phase, extending usually up to 6 months after the onset. The third phase, called the chronic phase, begins months to years after stroke, and it may continue for the rest of the person's life (Hillis et al., 2006; Yang et al., 2018). The mechanisms promoting the recovery and reorganization of language in post-stroke aphasia (PSA) are still unresolved. Until now, the role of the right hemisphere (RH) in the recovery of PSA remains controversial. Previous studies suggested an important role of RH in the recovery from PSA due to left hemisphere (LH) stroke (Barlow, 1877; Weiller et al., 1995; Musso et al., 1999; Abo et al., 2004; Winhuisen et al., 2005; Turkeltaub et al., 2012). Previous studies investigating treatment-induced neural plasticity in chronic aphasia showed increased RH activation associated with better recovery in the chronic stage (Elkana et al., 2013; Kiran et al., 2015). Some studies suggest that increased RH activation may result in excessive right-to-left suppression, which may hinder language performance (Belin et al., 1996; Heiss et al., 2003; Naeser et al., 2004; Thiel et al., 2006). Moreover, the relationship between structural changes in $\mathrm{RH}$ and lesions in $\mathrm{LH}$ remains unknown. Recently, neuroimaging has been suggested to play an important role in the exploration of the implication of RH in the recovery from PSA. For a description of specific language functions related to RH structures or brain networks, local gray matter volume (GMV) is commonly measured to explain additional variance in language outcome based on T1weighted images (Butler et al., 2014; Xing et al., 2016) along with white matter (WM) regions detected by diffusion tensor imaging (DTI) (Forkel et al., 2014; Ivanova et al., 2016; Pani et al., 2016).

Until now, several language models have been used to describe the relationship between language dysfunction and lesions in patients with PSA. The dual-stream model of language processing, one of the most used neuropsychological models of speech and language organization (Kreisler et al., 2000; Hickok and Poeppel, 2004, 2007; Fridriksson et al., 2016), has been used frequently as the classic template (Kümmerer et al., 2013; Fridriksson et al., 2016; Zündorf et al., 2016; Goucha et al., 2017; McKinnon et al., 2018; Northam et al., 2018) in order to evaluate language dysfunction concerning brain damage in PSA. This model describes two large-scale processing streams, namely, the dorsal stream is crucial for producing fluent speech and auditory-motor integration processes, and the ventral stream supports the mapping between sound and meaning and thus it is related to auditory comprehension (Saur et al., 2008; Chang et al., 2015; Sammler et al., 2015; Fridriksson et al., 2016; Garrod and Pickering, 2016). Most previous studies mainly focused on the location and extension of the brain damage after stroke, and less attention was paid to the differences in the native languages (Anglade et al., 2014). Tonal languages such as Mandarin differ from Indo-European languages in their morpho syllabic and segmental structure (Zhao et al., 2011; Leppänen et al., 2019). These differences between tonal and Indo-European languages have been evidenced not only in their linguistic features but also in their neural correlates (Wong et al., 2007) and even in genetic expression (Balter, 2006).

In this exploratory and prospective study, we investigated lesion distribution in Chinese patients with PSA in subacute phase with MRI and evaluated the language deficits from the prism of the dual-stream language model with the following purposes: (1) to study the lesion distribution and its relationship with specific language deficits in Chinese patients with PSA; (2) to explore whether the dual-stream model of Fridriksson et al. fits with the pattern of lesion deficit found in Chinese PSA and thus to conclude if the dual-stream model is valid to measure the language deficits observed in Chinese patients with PSA; and (3) to explore changes in RH of Chinese patients with PSA. To achieve these objectives, MRI analyses [e.g., voxel-based lesion-symptom mapping (VLSM), GMV analysis defined by Automated Anatomical Labeling (AAL) atlas, Tract-Based Spatial Statistics (TBSS), and tractography] were used.

\section{MATERIALS AND METHODS}

\section{Participants}

Consecutive 42 patients (female: 13 , male: 29 , mean age: $58 \pm 12$ ) with PSA were recruited between March 2014 and June 2018 from the neurology department of Dongzhimen Hospital, Speech and Language Therapy Center of China Rehabilitation Research Centre and Neurology Department of Peking University Third Hospital. Inclusion criteria were as follows: first-ever LH ischemic stroke that happened between 1 and 6 months before language testing and MRI examination; aphasia was confirmed by a research speech-language pathologist; and all patients were right-handed based on the Edinburgh Handedness Inventory (EHI) test since the EHI test has the advantage of being a simple and brief method of evaluating laterality using a quantitative scale (Veale, 2014) and the hemispheric lateralization was also assessed by using functional MRI according to a previous publication (Wilke and Lidzba, 2007). All patients were native Chinese speakers and monolinguals. The noninclusion criteria were as follows: history of another neurological or psychiatric disease; MRI-incompatible prosthesis; and inability to perform speech and language testing as well as MRI examinations due to the severity of aphasia or comorbidity. A control group composed of 30 healthy native Chinese-speaking controls (HC) matched by age, gender, and education level were included (Figure 1). All participants have signed an informed consent form before the start of the study. This study was approved by the Institutional Review Board and Ethics Committee of Beijing University of Chinese Medicine.

\section{Speech and Language Testing}

All patients performed a battery of language tests: Chinese Rehabilitation Research Center Standard Aphasia Examination (CRRCAE), which is designed according to the rules of Mandarin. The reliability and validity of this scale have been 
tested in a previous study and it has good reliability and sensitivity that can be used as a quantitative table for the diagnosis and treatment of aphasia among Mandarin users (Zhang et al., 2005; Tao et al., 2014; Zhu D. et al., 2014; Chang et al., 2017). According to the characteristics of aphasia symptoms, the scale was divided into three subitems, namely, comprehension, expression, and other speech-related abilities (e.g., calculation, copy, and cartoon description). In this study, comprehension was assessed at the reading and auditory levels, and production was measured through repetition and description levels.

\section{MRI Data Acquisition}

Different MRI sequences were acquired in all participants to obtain GM and WM information. Neuroimaging was acquired in a Siemens 3T Trio MRI scanner (Erlangen, Germany). Axial anatomical images were acquired using a high-resolution threedimensional T1-weighted images with the following: repetition time $(\mathrm{TR})=1,900 \mathrm{~ms}$, echo time $(\mathrm{TE})=2.13 \mathrm{~ms}$, time of inversion $(\mathrm{TI})=900 \mathrm{~ms}$, flip angle $=9^{\circ}$, resolution $=256 \times 256$, and voxel size $=1.0 \mathrm{~mm} \times 1.0 \mathrm{~mm} \times 1.0 \mathrm{~mm}$. DTI was acquired with a diffusion-weighted, single-shot, spin-echo, echo-planar imaging sequence using 30 directions with $b=0 \mathrm{~s} / \mathrm{mm}^{2}$ and $b=1,000 \mathrm{~s} / \mathrm{mm}^{2}$, slice thickness: $2 \mathrm{~mm}$, gap $=0 \mathrm{~mm}$, slices $=65$, TR: 11,000 ms, TE: $94 \mathrm{~ms}$, matrix: $128 \times 128$, field of view $($ FOV): $256 \times 256$, voxel size $=2 \times 2 \times 2$, and phase-encoding direction: $\mathrm{A}>>\mathrm{P}$.

\section{MRI Data Preprocessing}

\section{T1-Weighted Image}

T1-weighted imaging data preprocessing was carried out using $\mathrm{SPM}_{12}{ }^{1}$ and $\mathrm{CAT} 12^{2}$ with standard processing procedures including high-dimensional DARTEL (Diffeomorphic Anatomical Registration Through Exponentiated Lie Algebra) normalization algorithms and modulation for nonlinear components. Preprocessing steps also include the segmentation of whole-brain images into GM, WM, and cerebrospinal fluid and the normalization to the DARTEL template in MNI space (Template_1_IXI555_MNI152.nii). Finally, the volume of the 90 brain GM regions defined by the AAL atlas was calculated.

\section{Diffusion-Weighted Image}

Diffusion-weighted imaging data were preprocessed using FMRIB Software Library (FSL ${ }^{3}$ ) (Behrens et al., 2003, 2007). First, the correction for eddy currents and subject motion was applied using the "eddy_correct" function. Then, the volume with no diffusion weighting was extracted and named "nodif." Then, a single binarized volume in diffusion space containing ones inside the brain and zeroes outside the brain will be got by "bet2" in the "nodif." "dtifit" fitted a diffusion tensor model at each voxel. Outputs of dtifit have mean diffusivity (MD), fractional anisotropy (FA), first eigenvalue (L1), second eigenvalue (L2), and third eigenvalue (L3). In particular, L1 is also called axial

\footnotetext{
${ }^{1}$ https://www.fil.ion.ucl.ac.uk/spm/software/spm12/

${ }^{2}$ http://www.neuro.uni-jena.de/cat/

${ }^{3}$ http://www.fmrib.ox.ac.uk/fsl
}

diffusivity (AD), and the mean of $\mathrm{L} 1$ and $\mathrm{L} 2$ is called radial diffusivity (RD).

The TBSS pipeline was applied with recommended parameters. Voxel-wise statistical analysis of the FA data was carried out using TBSS, part of FSL. First, the FA data of all subjects were aligned into a common space using the FMRIB nonlinear image registration tool (FNIRT), which uses a b-spline representation of the registration warp field. Next, the mean FA image was created and thinned to create a mean FA skeleton, which represents the center of all tracts common to the group. The aligned FA data of each subject were then projected onto this skeleton and the resulting data were fed into voxel-wise cross-subject statistics (Rueckert et al., 1999; Smith, 2002; Smith et al., 2004; Smith et al., 2006).

We also obtained a WM fiber bundle that crosses the GM region in which there was a significant difference between the healthy control group and the patient group. WM fiber bundles were calculated with the TrackVis toolbox. ${ }^{4}$ Specifically, "dti_recon" was used to build the tensor, then "dti_tracker" was for deterministic fiber tracking. The default parameters or settings were performed. The termination conditions of fiber tracking include FA value $<0.2$ and deflection angle $>35^{\circ}$. We determined the WM fibers that passed through in native space, this gave fiber number (FN) maps. Referring to the abovementioned steps, the skeleton map of FN was generated.

\section{Lesion Identification}

Lesion-symptom mapping was demarcated on T1-weighted images manually using MRIcrogl ${ }^{5}$ in native space by neurologists who were blinded to the language scores of participants. Figure 2 shows a lesion overlap map for the aphasic participant group. The value of each voxel represented the number of patients with damaged at this voxel. The brighter the color in the figure, the more people injured in the area.

\section{Statistical Analysis}

\section{Voxel-Based Lesion-Symptom Mapping Analysis}

The purpose of VLSM analysis was used to identify the lesion area related to the language impairment. To better understand the effects of LH lesion on language performance, a VLSM was used to analyze the relationship between lesion and behavior (Bates et al., 2003) with VLSM toolbox ${ }^{6}$ based on MATLAB (R2016b, MathWorks, Massachusetts, MA, United States). VLSM analyses were run with 1,000 permutation tests resulting in T-maps that reflected critical regions where lesional tissue was associated with the performance on a given language measure. Significant results were derived from voxel-wise $t$-tests using a threshold of $p<0.05$ with permutation-based correction for multiple comparisons (Lukic et al., 2017).

\section{Gray Matter Volume Analysis}

The GMV analysis was used to evaluate the difference in the GM areas in $\mathrm{RH}$ between the patient group and the healthy

\footnotetext{
${ }^{4}$ http://trackvis.org/dtk/

${ }^{5}$ http://www.cabiatl.com/mricrogl/

${ }^{6}$ https://aphasialab.org/vlsm/
} 


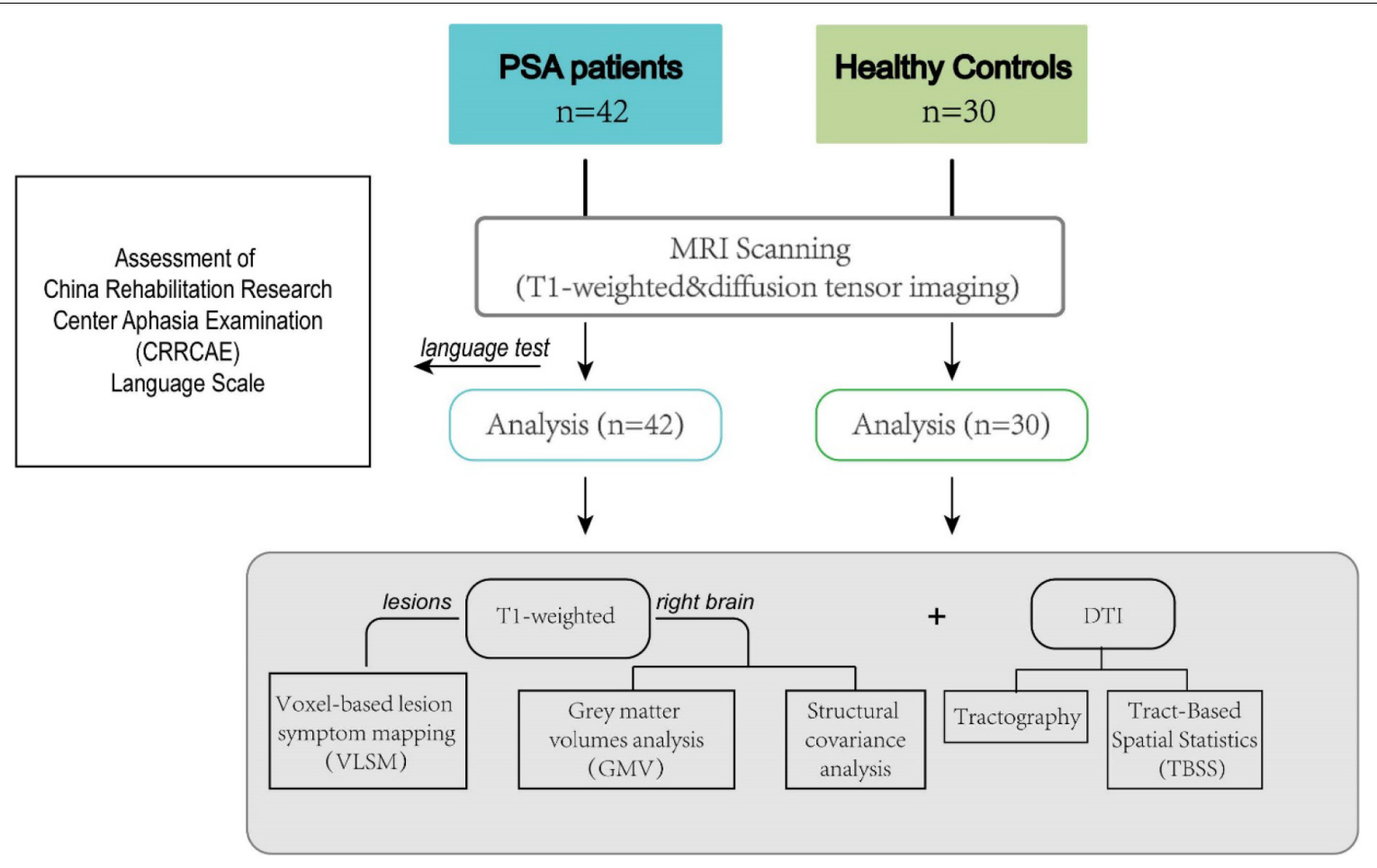

FIGURE 1 | Overview of the study design.

A

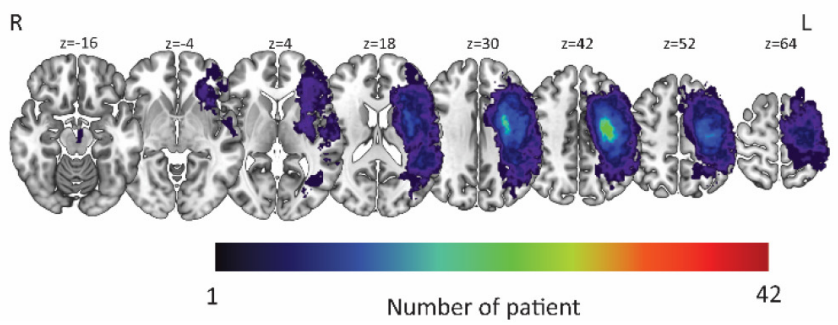

B

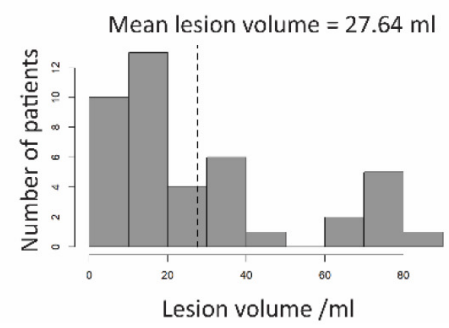

FIGURE 2 | Distribution of lesion in LH. (A) The lesion area overlap across patients was rendered on a brain template. The color bar represents the number of patients with damage in each voxel. Numbers on top of each axial map refer to the z-plane of the MNI space, respectively. (B) Distribution of the lesion volume across the patients. The average lesion volume was $27.64 \mathrm{ml}$. L, left hemisphere; R, right hemisphere.

control group. The group differences (healthy participant group vs. aphasic patient group) in GMV were examined in all regions of $\mathrm{RH}$, defined by the AAL atlas. For each region, the mean GMV was calculated. A linear regression was also used to access whether there were differences between the groups. The results were adjusted for the 45 regions with the Bonferroni correction method (corrected $p<0.05$ ). In addition, we also examined the difference in the whole-brain, $\mathrm{LH}$, and RH GMV between the healthy control group and the aphasic patient group.

Structural covariance between the left lesion regions and the right significant regions was assessed. Using Pearson's correlation coefficient, the covariance between the brain regions was defined. Specifically, the co-degeneration between the volume of the brain area with a difference in the right brain and the volume of the left brain area determined by VLSM was calculated, and $p<0.05$ was taken as the criterion for judging significance.

\section{Tract-Based Spatial Statistics Analysis}

The TBSS analysis was used to evaluate the difference of WM in $\mathrm{RH}$ between patients and healthy controls. First, we examined the relationship of FA, a measure for fiber density, axonal diameter, and myelination in WM between groups in skeleton regions of $\mathrm{RH}$. A design matrix was generated using the "design_ttest2" in FSL. The FA maps were categorized as controls or patients. Statistical analysis was performed using the FSL tool "randomize" with 1,000 permutations. Threshold-Free Cluster Enhancement (TFCE; Smith and Nichols, 2009) was applied for correction of multiple comparisons. The threshold for statistical significance 
was $p<0.05$. Then, the mean FA was calculated in significant skeleton regions. Other measures of $\mathrm{WM}$, such as $\mathrm{MD}, \mathrm{AD}$, and $\mathrm{RD}$, were also processed in the same way. Using the linear model, the relationship between the index on the WM skeleton and the language performance in the WM fiber defined in the Johns Hopkins University (JHU) template was calculated.

\section{Tractography Analysis}

Tractography was used to evaluate the differences of WM fibers associated with different GM regions between the patient group and the healthy control group. For tractography, the mean FN, $\mathrm{FA}, \mathrm{MD}, \mathrm{AD}$, and $\mathrm{RD}$ of all subjects were calculated in the $\mathrm{FN}$ skeleton map. Then, we examined the differences of those indexes between the healthy control and patients with PSA by a twosample $t$-test. In addition, the relationship between the volume of brain regions with differences in volume between the control group and the PSA group and the WM fiber indicators passing through these brain regions was calculated.

\section{RESULTS}

\section{Demographic and Behavioral Results}

Patients with PSA and $\mathrm{HC}$ did not significantly differ in age, gender, or years of education. Stroke-related clinical characteristics of the patients were tested using the National Institutes of Health Stroke Scale (NIHSS) (Lyden et al., 2001; Cooray et al., 2015). The overall production and comprehension ability were evaluated by $z$ scores of their subitems: Listen,

TABLE 1 | Demographic and behavioral results.

\begin{tabular}{|c|c|c|c|c|c|}
\hline & Total & $\mathrm{HC}$ & PSA & $t / \chi^{2}$ & $p$ \\
\hline$n$ & 72 & 30 & 42 & - & - \\
\hline Age (years) & $58 \pm 12$ & $56 \pm 11$ & $58 \pm 12$ & 64.08 & 0.468 \\
\hline Gender (F/F\%) & $22 / 31 \%$ & $9 / 30 \%$ & $13 / 31 \%$ & 0.007 & 0.931 \\
\hline Education (years) & $10 \pm 4$ & $10 \pm 4$ & $10 \pm 3$ & -0.124 & 0.901 \\
\hline Handedness (R/L) & $72 / 0$ & $30 / 0$ & $42 / 0$ & - & - \\
\hline Time post stroke (days) & - & - & $81 \pm 70$ & - & - \\
\hline Lesion volume (ml) & - & - & $28 \pm 25$ & - & - \\
\hline TIV (ml) & 1490123 & 1483150 & 1494115 & -0.354 & 0.725 \\
\hline GM volume (ml) & $548 \pm 61$ & $581 \pm 60$ & $524 \pm 61^{\star \star \star}$ & 3.954 & $<0.001$ \\
\hline WM volume (ml) & $487 \pm 54$ & $508 \pm 59$ & $472 \pm 57^{\star}$ & 2.589 & 0.012 \\
\hline CSF volume (ml) & $450 \pm 89$ & $392 \pm 61$ & $491 \pm 84^{\star \star \star}$ & -5.497 & $<0.001$ \\
\hline Left GM volume (ml) & $212 \pm 30$ & $232 \pm 25$ & $198 \pm 29^{\star \star \star}$ & 5.158 & $<0.001$ \\
\hline Right GM volume (ml) & $221 \pm 24$ & $231 \pm 24$ & $214 \pm 24^{\star \star}$ & 2.899 & 0.005 \\
\hline Comprehension Z-score & - & - & $0 \pm 0.95$ & - & - \\
\hline Production Z-score & - & - & $0 \pm 0.94$ & - & - \\
\hline Listen & - & - & $16 \pm 12$ & - & - \\
\hline Read & - & - & $16 \pm 12$ & - & - \\
\hline Repeat & - & - & $10 \pm 9$ & - & - \\
\hline Speak & - & - & $7 \pm 9$ & - & - \\
\hline NIHSS & - & - & $7 \pm 4$ & - & - \\
\hline
\end{tabular}

HC, healthy control; PSA, post-stroke aphasia; TIV: total intracranial volume; GM: gray matter volume; WMV, white matter volume; CSF, cerebrospinal fluid. ${ }^{*} p<0.05 ;{ }^{* *} p<0.01 ;{ }^{* * *} p<0.001$, versus $H C$.
TABLE 2 | Overlap between lesion areas and the dual-stream model.

\begin{tabular}{|c|c|c|c|}
\hline Region & Abbreviation & Dorsal & Ventral \\
\hline Angular gyrus & ANG & & Yes \\
\hline Caudate nucleus & CAU & & Yes \\
\hline Inferior frontal gyrus, opercular part & IFGoperc & Yes & \\
\hline Inferior frontal gyrus, orbital part & ORBinf & Yes & Yes \\
\hline Inferior frontal gyrus, triangular part & IFGtriang & Yes & \\
\hline Insula & INS & Yes & Yes $(0.0048)$ \\
\hline Middle frontal gyrus & MFG & Yes $(0.0537)$ & \\
\hline Middle occipital gyrus & MOG & & Yes \\
\hline Lenticular nucleus, pallidum & PAL & & \\
\hline Postcentral gyrus & PoCG & Yes $(0.1163)$ & \\
\hline Precentral gyrus & PreCG & Yes $(0.4072)$ & \\
\hline Lenticular nucleus, putamen & PUT & Yes & Yes \\
\hline Supramarginal gyrus & SMG & Yes & Yes \\
\hline Inferior temporal gyrus & ITG & & Yes \\
\hline Middle temporal gyrus & MTG & & Yes \\
\hline Temporal pole: middle temporal gyrus & TPOmid & & Yes \\
\hline Temporal pole: superior temporal gyrus & TPOsup & & Yes \\
\hline Superior temporal gyrus & STG & & Yes \\
\hline
\end{tabular}

The values in brackets in the dorsal and ventral columns represent the overlap rate between the VLSM result and the dual-stream model raised by Fridriksson et al. (2016).

Read, Repeat, and Speak. (The results showed that these patients had functional impairment in Listen, Read, Repeat, and Speak. Among these results, the average scores of the patients for the four tests were $16 \pm 12,16 \pm 12,10 \pm 9$, and $7 \pm 9$, out of 40,40 , 30 , and 30, respectively; Table $\mathbf{1}$ ).

\section{Voxel-Based Lesion-Symptom Mapping Results}

As shown in Figure 3, the comprehension- and productionrelated lesions were derived from VLSM analysis. There was a negative correlation between production ability and lesions in the precentral, superior frontal, middle frontal, and postcentral gyri of LH $(p=0.001)$. Similarly, the comprehension ability was negatively correlated with lesions in the precentral gyrus, superior frontal gyrus, middle frontal gyrus, insula gyrus, postcentral gyrus, and inferior parietal lobule of LH $(p<0.001)$. It is worth mentioning that the left precentral, superior frontal, middle frontal, and postcentral gyri showed a negative correlation with the performance of both comprehension and production abilities.

These resulting clusters (Table 2) were compared with areas comprised in the dorsal and ventral streams in the dual-stream model, as reported in the study by Fridriksson et al. (2016). Our results showed clear differences in lesion areas between the description of Fridriksson et al.'s dualstream model and that of this study. The lesion areas in our results were consistent neither with the description of lesion areas in the superior frontal and middle frontal of the dorsal pathway by Fridriksson et al. nor with the lesion areas in precentral, superior frontal, middle frontal, postcentral, and inferior parietal lobule of the ventral stream (Figure 3E). 
A
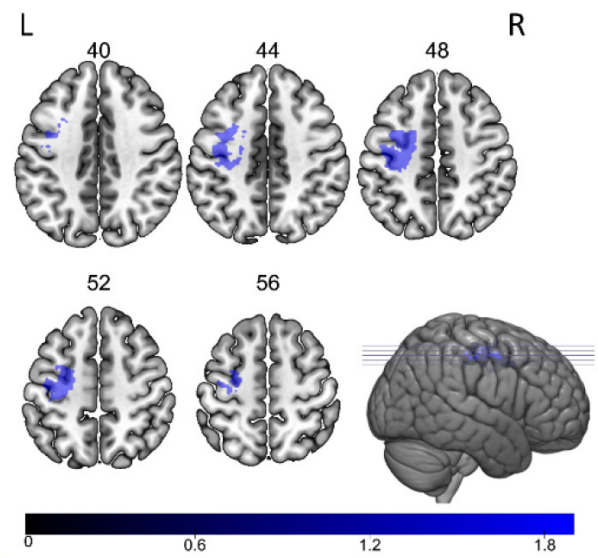

Production

C
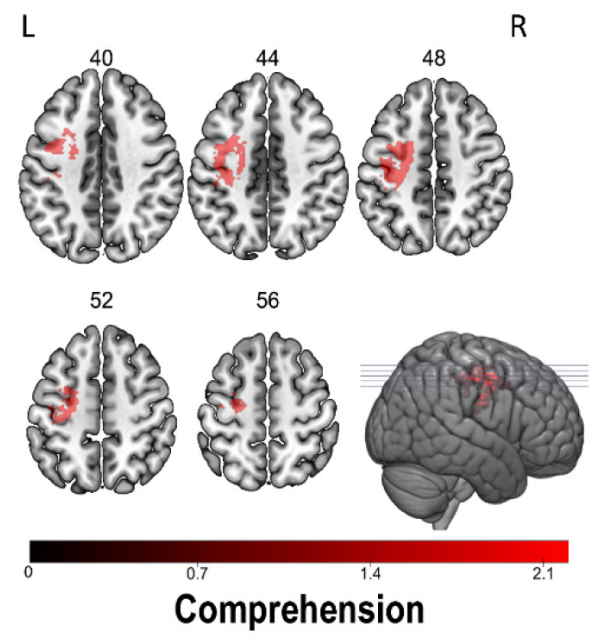

E

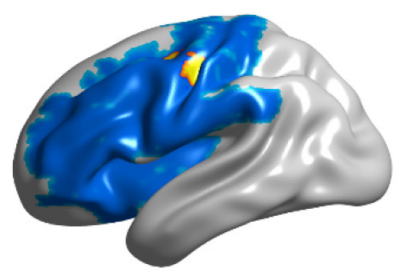

B

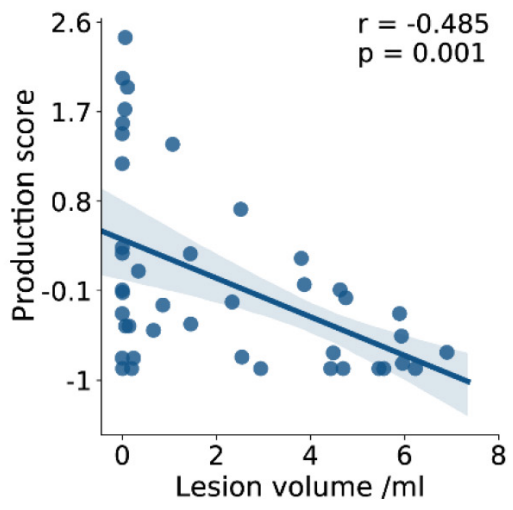

D
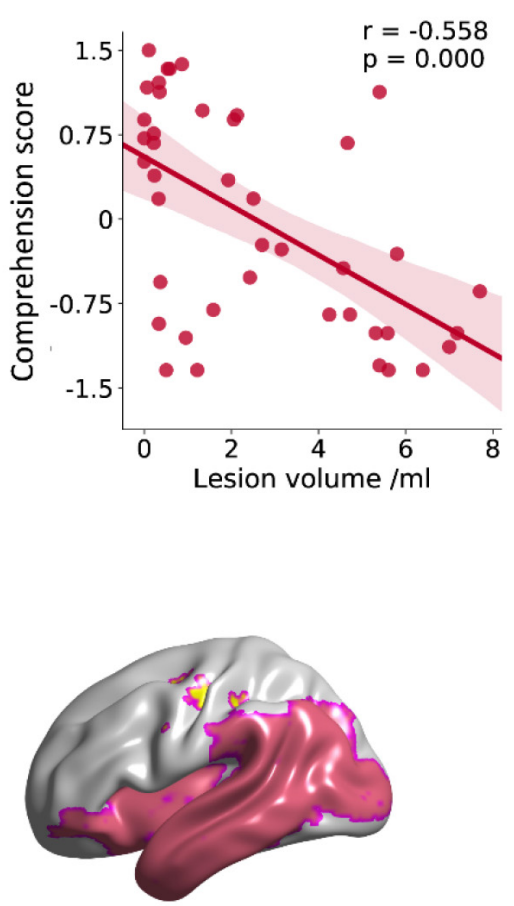
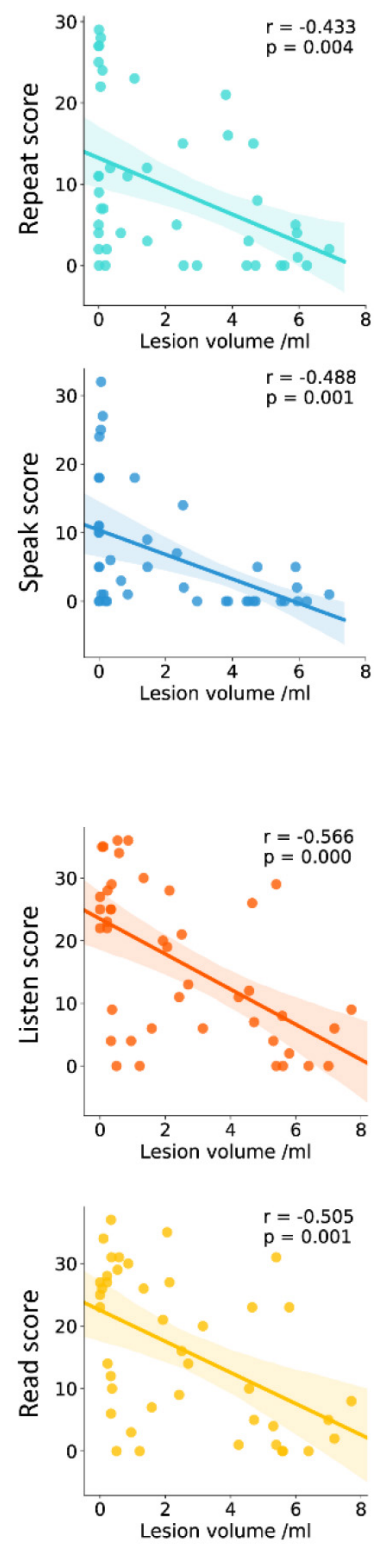

Overlap between production-related lesions and the dorsal stream

Overlap between comprehension-related lesions and the ventral stream

FIGURE 3 | Lesion areas related to linguistic score. (A,C) A negative correlation was found between gray matter volume and production in the left frontoparietal cortex (cluster size $=894$, peak coordinates: $x=-28, y=-16, z=50, t=3.74$, Benjamini-Hochberg correction) and between comprehension in parietal cortex and insula gyrus (cluster size $=1,046$, peak coordinates: $x=-26, y=-26, z=48, t=4.41$, Benjamini-Hochberg correction). Numbers on top of each axial map refer to the $z$-plane of the MNI space. (B,D) Scatter plot showing partial regression using language scores as the dependent measure and gray matter extractions of cluster as the independent measure. These correlations did not differ significantly after controlling for covariates of no interest such as age, gender, the level of education, and handedness. (E) The overlap rates of language-related lesions (yellow), with dorsal pathway (blue) and ventral pathway (pink) of $57.72 \%$ ( $57.72 \%$ of all production-related lesions located in the dorsal pathway) and $0.48 \%$ ( $0.48 \%$ of all comprehension-related lesions located in the ventral pathway), respectively. Rate $=$ overlapping part with dual-stream/lesion areas. 
A

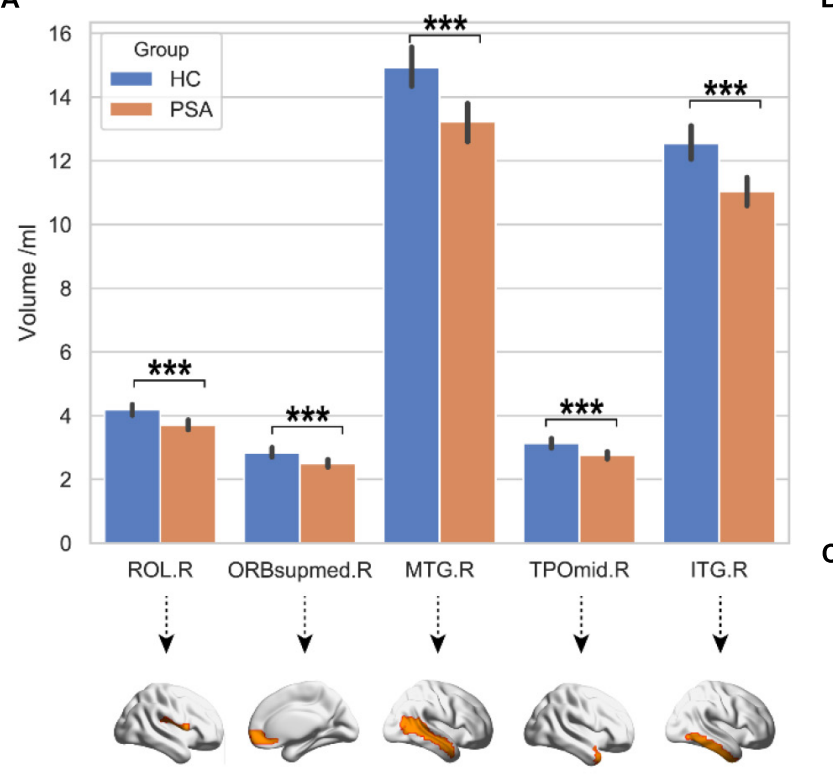

Cortical Regions
B

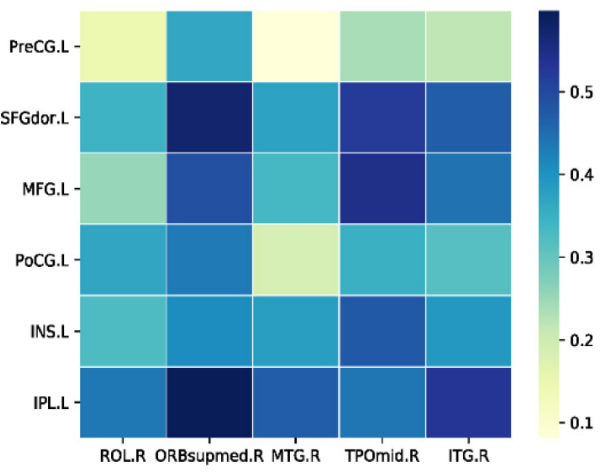

C

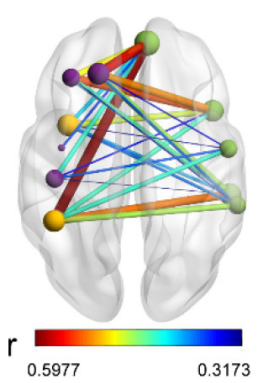

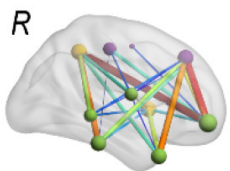

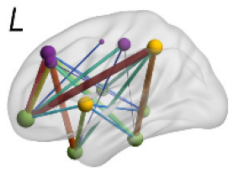

FIGURE 4 | Decreased GMV in the right hemisphere. (A) The GMV in RH of PSA group was significantly lower than that of HC group. (B,C) The maps illustrate results of structural covariance analysis between language-related lesions and significantly decreased GM in RH. The color represents the size of $r$ value. ROL, rolandic oper; ORBsupmed, superior frontal gyrus (medial orbital); MTG, middle temporal gyrus; TPOmid, temporal pole, middle temporal gyrus; ITG, inferior temporal gyrus; PreCG, precentral gyrus; SFGdor, superior frontal gyrus (dorsolateral); MFG, middle frontal gyrus; INS, insula; PoCG, postcentral gyrus; IPL, inferior parietal. Letters $L$ and $R$ in (C) correspond to the left and right hemispheres, respectively. ${ }^{* \star *} P<0.001$.

Lesion overlapping for all patients is shown in Figure 3E. The overlap rates of language-related lesions (yellow) with a dorsal pathway (blue), and ventral pathway (pink) were $57.72 \%$ of all production-related lesions located in the dorsal pathway and only $0.48 \%$ of all comprehension-related lesions located in the ventral pathway.

TABLE 3 | White matter indexes in patients with PSA compared with HC.

\begin{tabular}{|c|c|c|c|c|c|c|}
\hline & \multicolumn{2}{|c|}{$\mathrm{HC}$} & \multicolumn{2}{|c|}{ PSA } & \multicolumn{2}{|c|}{ Test } \\
\hline & Mean & SD & Mean & SD & $t$ & $p$ \\
\hline \multicolumn{7}{|c|}{ Indexes in skeleton by TBSS } \\
\hline FA values $\left(\times 10^{-1}\right)$ & 7.039 & 0.156 & 6.400 & 0.577 & 5.826 & $<0.001$ \\
\hline MD values $\left(\times 10^{-4}\right)$ & 3.642 & 0.154 & 4.412 & 0.938 & -4.387 & $<0.001$ \\
\hline$A D$ values $\left(\times 10^{-4}\right)$ & 7.108 & 0.211 & 8.066 & 1.059 & -4.816 & $<0.001$ \\
\hline RD values $\left(\times 10^{-4}\right)$ & 1.899 & 0.156 & 2.664 & 0.878 & -4.649 & $<0.001$ \\
\hline \multicolumn{7}{|c|}{ Indexes in fiber by fiber tracking } \\
\hline FN & 5.241 & 1.434 & 6.305 & 3.396 & -1.592 & 0.116 \\
\hline FA values $\left(\times 10^{-1}\right)$ & 5.330 & 0.220 & 5.070 & 0.574 & 2.320 & 0.023 \\
\hline MD values $\left(\times 10^{-4}\right)$ & 4.285 & 0.172 & 4.703 & 0.846 & -2.626 & 0.011 \\
\hline AD values $\left(\times 10^{-4}\right)$ & 6.837 & 0.259 & 7.284 & 0.837 & -2.784 & 0.007 \\
\hline RD values $\left(\times 10^{-4}\right)$ & 2.974 & 0.171 & 3.386 & 0.861 & -2.546 & 0.013 \\
\hline
\end{tabular}

\section{Gray Matter Volume in the Right Hemisphere of Post-stroke Aphasia}

The PSA group showed a significantly lower mean GMV than HC group $(p<0.001)$, as shown in Figure 4A. Meanwhile, a structural covariance was found between the declined GM areas and language-related lesions in LH (Figure 4B). The superior frontal gyrus, medial orbital, and middle and inferior temporal gyri were key nodes with higher weight in structural covariance (Figure 4C).

\section{White Matter Structure in Right Hemisphere}

The TBSS results, as shown in Table 3, indicated lower integrity in the tracts of the PSA group as compared with those of HC. There were significant differences in $\mathrm{FA}$ values and $\mathrm{MD}, \mathrm{AD}$, and $\mathrm{RD}$ values of RH between the PSA group and the HC group. A significant decrease in FA values and a significant increase in $\mathrm{MD}, \mathrm{AD}$, and $\mathrm{RD}$ values were demonstrated in patients with PSA as compared with HC (Table 3). Five clusters showed a positive correlation with language abilities in patients with PSA (Figure 5).

Deterministic tractography analyses revealed, as shown in Figure 6A, a positive correlation between GMV and FA in the tracts passing through these areas in RH. Figures $6 \mathbf{B}, \mathbf{C}$ demonstrates that reduced GMV was also associated 


\section{Anterior thalamic radiation $\mathrm{R}$}

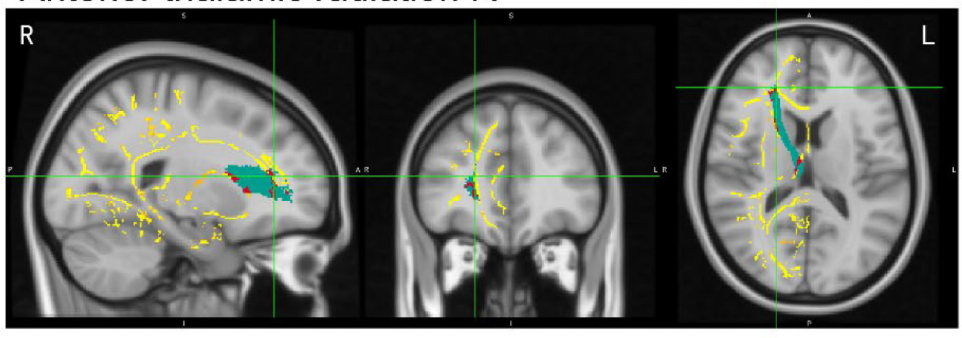

Corticospinal tract $\mathrm{R}$

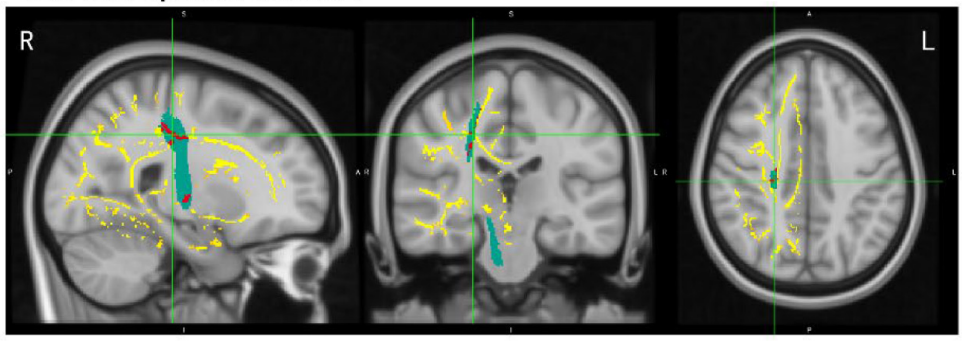

Cingulum (hippocampus) $\mathrm{R}$

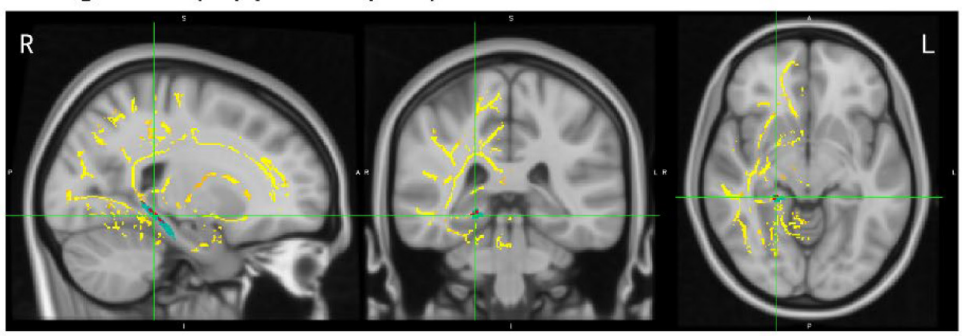

Superior longitudinal fasciculus $\mathrm{R}$

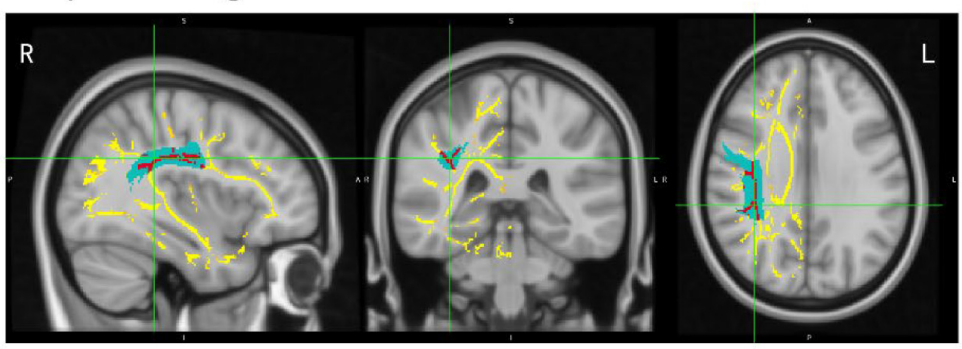

Cingulum (hippocampus) $\mathrm{R}$

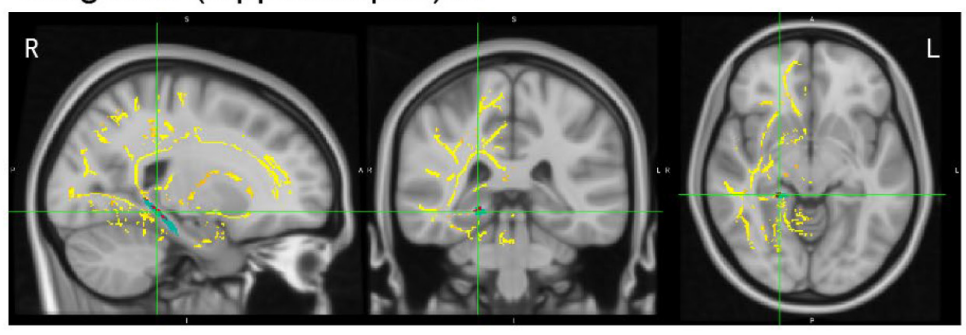

ATR. R with skeleton

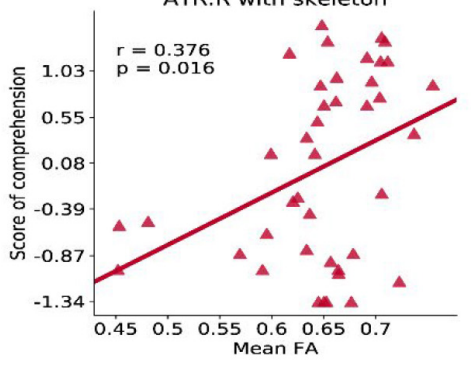

CST.R with skeleton

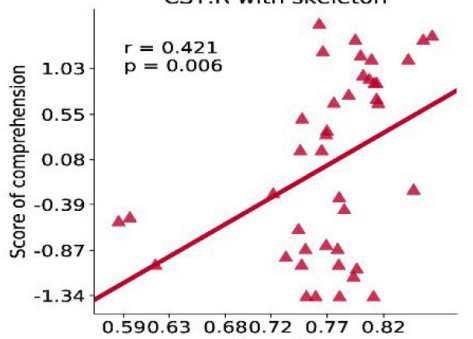

Mean FA

CgH.R with skeleton

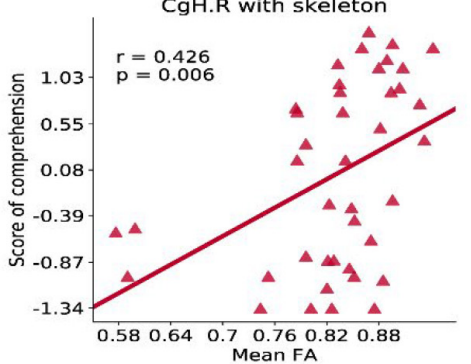

SLF.R with skeleton

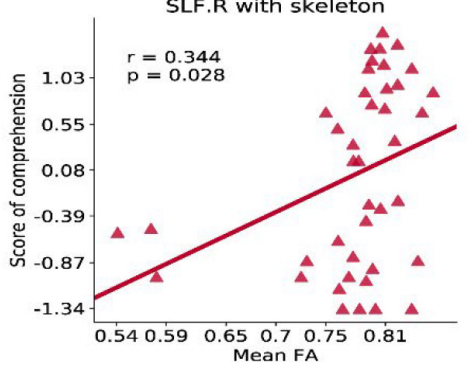

$\mathrm{CgH} . \mathrm{R}$ with skeleton

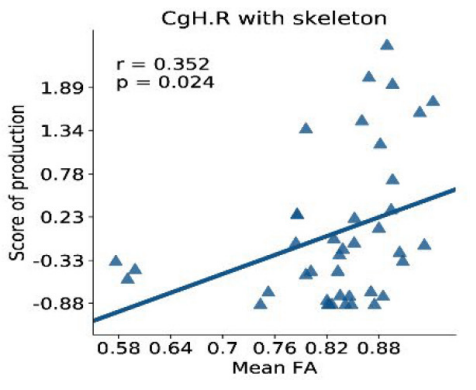

FIGURE 5 | TBSS map in RH of PSA related to linguistic score. The mean FA template produced by TBSS pipeline, overlaid (axial, coronal, and sagittal view) with the skeleton generated using an FA threshold of 0.2. We do TBSS analysis in RH searching for the differences between the PSA group and the HC group $(p<0.05$, TFCE-corrected). Red areas indicate regions with significantly reduced FA values in the PSA group compared with $\mathrm{HC}$. Scatter maps represent ROI areas significantly related to the comprehension (red) or production (blue). FA, fractional anisotropy; TBSS, Tract-Based Spatial Statistics; TFCE, threshold-free cluster enhancement. 


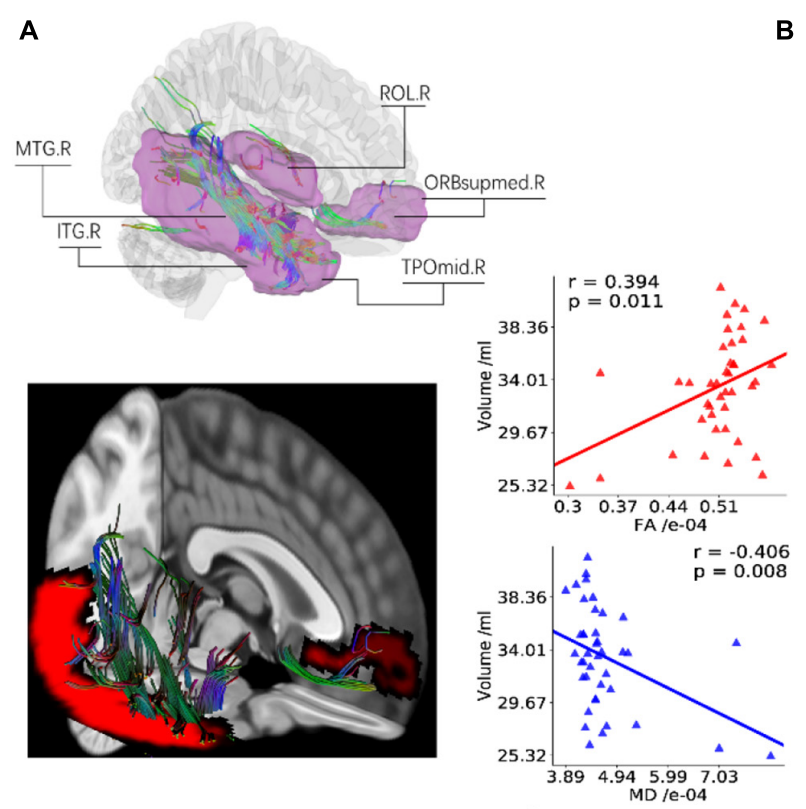

Fiber tracking betweenn ROI
B

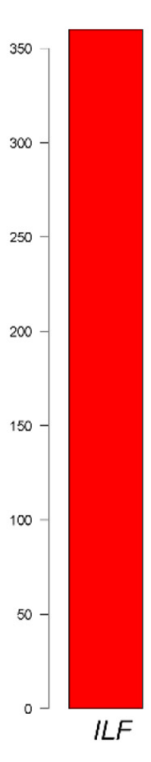

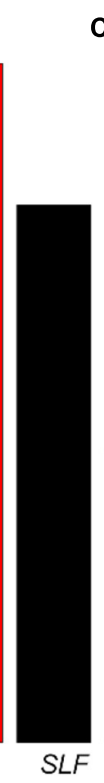

C
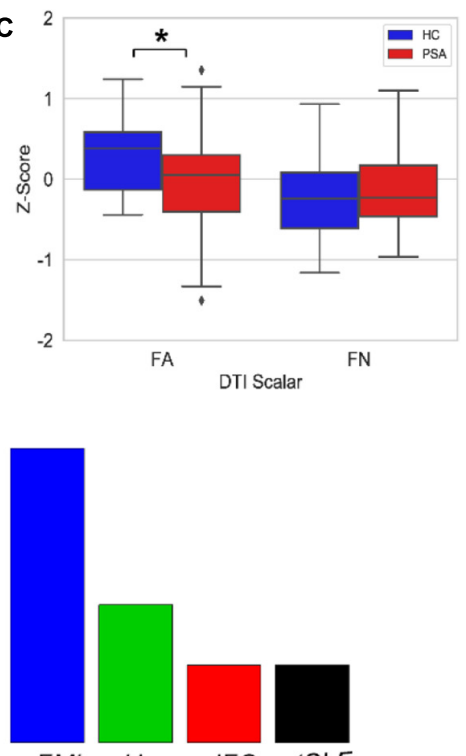

FM
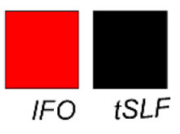

WM voxel

FIGURE 6 | White fiber connecting reduced GM regions in $\mathrm{RH}$. (A) All structural covariant regions (pink) in $\mathrm{RH}$ and WM fibers cross them are tracked by deterministic tracking. The scatter plots showed correlations between FAMD and GMV. (B) The bar plot displays the voxel number of WM constructed from fiber tracing overlapped with Johns Hopkins University (JHU) WM atlas. (C) Box plots present differences in WM indexes between healthy people and patients. F6A is a schematic diagram of the fiber tracking. The purple area indicates the differences in the right hemisphere area between the healthy control group and the patient with PSA group. F6B represents the overlap between the fiber skeleton obtained by fiber tracking and the area defined by the WM template, which is used to determine the spatial position of the fiber. F6C represents the difference between FN and FA between the healthy control group and the patient with PSA group. ROL, rolandic oper; ORBsupmed, superior frontal gyrus (medial orbital); MTG, middle temporal gyrus; TPOmid, temporal pole middle temporal gyrus; ITG, inferior temporal gyrus; ILF, inferior longitudinal fasciculus; SLF, superior longitudinal fasciculus; FMi, forceps minor; Unc, uncinate fasciculus; IFO, inferior fronto-occipital fasciculus; tSLF, superior longitudinal fasciculus (temporal part); FA, fractional anisotropy; FN, fiber number. ${ }^{*} p<0.05$.

with reduced FA in WM linking them while FN did not change significantly.

\section{DISCUSSION}

Our previous study has shown that the damage areas in post-stroke motor aphasia were not only located in the areas of the well-known motor speech center such as the Broca's area, but some other damaged areas might be also involved in the formation of motor aphasia in Chinese patients with PSA (Chang et al., 2017). In this study, we found that lesions in sensorimotor areas are highly correlated with language comprehension and expression in Chinese native speakers. The present results are consistent with a previous study reporting that the human brain sensorimotor system may play an important role in the language process (Buchsbaum and D'Esposito, 2019).

Fridriksson et al. (2016) used the dual-stream model among English-speaking patients with PSA and demonstrated that distinct anatomical boundaries were revealed between a dorsal frontoparietal stream (form-to-articulation pathway) and a ventral temporal-frontal stream (form-to-meaning pathway), showing a division between two processing routes underlying English speech processing (Fridriksson et al., 2016). Previous studies performed by Ivanova et al. (2016) in native Russiaspeaking patients with PSA and Rosso et al. (2015) in native French-speaking patients with PSA have shown some variabilities in the results as compared with the study results in English speakers (Hickok and Poeppel, 2004; Forkel et al., 2014), suggesting that different native languages might influence the dual-stream model although English, French, and Russian belong to a family of Indo-European languages.

Previous study (Valaki et al., 2004) has shown that tonal language such as Chinese differs from Indo-European languages not only in its written and spoken forms but also in the cerebral mechanisms involved in language functions. Valaki et al. (2004) found that while Indo-European language (e.g., Spanish and English) speakers showed strong lateralization of activity sources to $\mathrm{LH}$, the Chinese demonstrated greater individual variability in the degree and direction of hemispheric asymmetries with emerging a bilaterally symmetric profile. These differences in the degree of hemispheric asymmetry were primarily due to a greater degree of activation in the right temporoparietal region in the Chinese group, suggesting increased participation of $\mathrm{RH}$ (Valaki et al., 2004).

In this study, lesions in sensorimotor areas correlated with both comprehension and production abilities in Chinese patients with PSA. A lesion-related language model was used in this study to distinguish the pathways of comprehension and production 
in native Chinese-speaking patients. The sensorimotor area and most of the frontal lobe are involved in lesion model response for both production and comprehension. We compared this model with the dual-stream model of Fridriksson et al. by calculating the overlap rate. The results showed only $0.5 \%$ of lesions related to comprehension overlap with the ventral stream, which is responsible for the meaning of language, and about $50 \%$ of lesions related to production overlap with the dorsal stream, which is responsible for expression. Furthermore, brain regions related to Chinese and Western language pathways have been reported to be different (Bolger et al., 2005; Tan et al., 2005). Previous studies have also shown that Chinese patients with PSA and English-speaking patients with PSA had different languagerelated GM areas (Bolger et al., 2005; Tan et al., 2005) and different WM clusters (Zhu L. et al., 2014). For the first time, to the best of our knowledge, we demonstrate in this study that Chinese patients with PSA and English-speaking patients with PSA may have similarities in production-related lesions but may be quite different in comprehension-related lesions. Our results suggested that the dual-stream model of Fridriksson et al. may not be suitable for the evaluation of the language abilities in Chinese patients with PSA in the subacute phase of recovery. Therefore, a language model should be established in the patient's native language.

In this prospective study, a significantly structural covariance was found between reduced GMV in $\mathrm{RH}$ and language-related lesions in LH. Meanwhile, the TBSS skeleton of the PSA group showed decreased FA and increased MD when compared with $\mathrm{HC}$ groups, thus reflecting a decreased integrity of WM in $\mathrm{RH}$ after LH stroke. The fiber tracking through reduced GM areas also decreased in FA values with no difference in $\mathrm{FN}$, indicating that the WM integrity in these areas was also affected. Previous studies have shown a compensatory increase of GMV in $\mathrm{RH}$ during recovery of PSA (Crinion and Price, 2005; Forkel et al., 2014; Lukic et al., 2017). However, our results demonstrated that GMV in RH significantly decreased in Chinese patients with PSA. One of the explanations for this difference may be that previous studies were done in patients who had stroke for more than 6 months before, whereas in this study, patients were included between 1 and 6 months after stroke. Another possible explanation is that there may be a difference not only in lesion distribution but also in RH structure alteration after $\mathrm{LH}$ stroke between Chinese patients with PSA and English-speaking patients with PSA due to different language mechanisms.

There are some limitations of this study. The first limitation is the heterogeneity in the inclusion time. Only patients in the subacute phase of recovery (between 1 and 6 months poststroke) were included in this study. Previous study (Saur et al., 2006) has shown a difference in overall patterns of language activation for different phases after stroke. Saur et al. (2006) found a large increase of activation in the bilateral language network with the strongest increase of activation in $\mathrm{RH}$ language areas during the subacute phase, which was different from the pattern of language activation in the chronic phase. The imaging parameters including GMV may change during this time. Therefore, the results of patients at the beginning of subacute phase (e.g., 1-month post-stroke) may be different from those of patients 6 months post-stroke. However, most patients with PSA were included in this study between 2 and 3 months (81 \pm 70 days) post-stroke; therefore, the heterogeneity of our study was relatively small. It will be interesting to know the status of GMV in RH more than 6 months after stroke in Chinese patients with PSA. Moreover, only English-speaking patients with PSA in the chronic phase ( $\geq 6$ months after the onset of stroke) were included in the dual-stream model study by Fridriksson et al. (2016); therefore, this might explain partly why the dualstream model of Fridriksson et al. may not be suitable for the evaluation of the language abilities in Chinese patients with PSA in the subacute phase of recovery. Second, Western languagespeaking patients with PSA were not directly included in this study. Third, this study has shown that only a small number of patients had the lesions distributed in the ventral pathway. The muster of lesions may be typical for Chinese native speaker patients with PSA. However, further study with a larger number of patients is needed for verification of the results.

\section{CONCLUSION}

In this prospective study, we investigated lesion distribution and early changes of $\mathrm{RH}$ in Chinese patients with PSA. Our results suggested for the first time that the dual-stream model of Fridriksson et al. is not suitable for the language evaluation of Chinese patients with PSA in the subacute phase of recovery. Furthermore, decreased GMV and low WM integrity were found in Chinese patients with PSA compared with HC.

\section{DATA AVAILABILITY STATEMENT}

The raw data supporting the conclusions of this article will be made available by the authors, without undue reservation.

\section{ETHICS STATEMENT}

The studies involving human participants were reviewed and approved by the Institutional Review Board and Ethics Committee of Dongzhimen hospital affiliated to Beijing University of Chinese Medicine. The patients/participants provided their written informed consent to participate in this study. Written informed consent was obtained from the individual(s) for the publication of any potentially identifiable images or data included in this article.

\section{AUTHOR CONTRIBUTIONS}

All authors listed have made a substantial, direct, and intellectual contribution to the work, and approved it for publication.

\section{FUNDING}

This study was supported by the Beijing Natural Science Foundation (project no. 7181005), the University Medicine 
Seed Fund for Interdisciplinary Research (grant no. BMU2018MX027), and the Research Funds for the Central Universities (project no. 2019-JYB-TD-003). The research leading to these results has received funding from the National Science Foundation of China (project no. 81473654) and the Special Public Welfare Industry and Scientific Research from the State Administration of Traditional Chinese Medicine (project no. 201407001-9).

\section{REFERENCES}

Abo, M., Senoo, A., Watanabe, S., Miyano, S., Doseki, K., Sasaki, N., et al. (2004). Language-related brain function during word repetition in post-stroke aphasics. Neuroreport 15, 1891-1894. doi: 10.1097/00001756-200408260-00011

Anglade, C., Thiel, A., and Ansaldo, A. I. (2014). The complementary role of the cerebral hemispheres in recovery from aphasia after stroke: a critical review of literature. Brain Inj. 28, 138-145. doi: 10.3109/02699052.2013.859734

Balter, M. (2006). Bruce Lahn profile. Links between brain genes, evolution, and cognition challenged. Science 314, 1872. doi: 10.1126/science.314.5807.1872

Barlow, T. (1877). On a Case of Double Hemiplegia, with Cerebral Symmetrical Lesions. Br Med J. 2, 103-104. doi: 10.1136/bmj.2.865.103

Bates, E., Wilson, S. M., Saygin, A. P., Dick, F., Sereno, M. I., Knight, R. T. et al. (2003). Voxel-based lesion-symptom mapping. Nat. Neurosci. 6, 448-450. doi: $10.1038 / \mathrm{nn} 1050$

Behrens, T. E., Berg, H. J., Jbabdi, S., Rushworth, M. F., and Woolrich, M. W. (2007). Probabilistic diffusion tractography with multiple fibre orientations: What can we gain? Neuroimage. 34, 144-155. doi: 10.1016/j.neuroimage.2006. 09.018

Behrens, T. E., Woolrich, M. W., Jenkinson, M., Johansen-Berg, H., Nunes, R. G., Clare, S., et al. (2003). Characterization and propagation of uncertainty in diffusion-weighted MR imaging. Magn Reson Med . 50, 1077-1088. doi: 10. 1002/mrm.10609

Belin, P., Van Eeckhout, P., Zilbovicius, M., Remy, P., François, C., Guillaume, S., et al. (1996). Recovery from nonfluent aphasia after melodic intonation therapy: a PET study. Neurology . 47, 1504-1511. doi: 10.1212/wnl.47.6.1504

Bolger, D. J., Perfetti, C. A., and Schneider, W. (2005). Cross-cultural effect on the brain revisited: universal structures plus writing system variation. Hum Brain Mapp . 25, 92-104. doi: 10.1002/hbm.20124

Buchsbaum, B. R., and D'Esposito, M. (2019). A sensorimotor view of verbal working memory. Cortex. 112, 134-148. doi: 10.1016/j.cortex.2018.11.010

Butler, R. A., Lambon, Ralph MA, and Woollams, A. M. (2014). Capturing multidimensionality in stroke aphasia: mapping principal behavioural components to neural structures. Brain . 137, 3248-3266. doi: 10.1093/brain/awu286

Chang, E. F., Raygor, K. P., and Berger, M. S. (2015). Contemporary model of language organization: an overview for neurosurgeons. J. Neurosurg. 122, 250-261. doi: 10.3171/2014.10.Jns132647

Chang, J., Zhang, H., Tan, Z., Xiao, J., Li, S., and Gao, Y. (2017). Effect of electroacupuncture in patients with post-stroke motor aphasia : Neurolinguistic and neuroimaging characteristics. Wien. Klin. Wochenschr. 129, 102-109. doi: 10.1007/s00508-016-1070-1

Cooray, C., Fekete, K., Mikulik, R., Lees, K. R., Wahlgren, N., and Ahmed, N. (2015). Threshold for NIH stroke scale in predicting vessel occlusion and functional outcome after stroke thrombolysis. Int J Stroke. 10, 822-829. doi: 10.1111/ijs.12451

Crinion, J., and Price, C. J. (2005). Right anterior superior temporal activation predicts auditory sentence comprehension following aphasic stroke. Brain . 128, 2858-2871. doi: 10.1093/brain/awh659

Elkana, O., Frost, R., Kramer, U., Ben-Bashat, D., and Schweiger, A. (2013). Cerebral language reorganization in the chronic stage of recovery: a longitudinal fMRI study. Cortex. 49, 71-81. doi: 10.1016/j.cortex.2011.09.001

Engelter, S. T., Gostynski, M., Papa, S., Frei, M., Born, C., Ajdacic-Gross, V., et al. (2006). Epidemiology of aphasia attributable to first ischemic stroke: incidence, severity, fluency, etiology, and thrombolysis. Stroke . 37, 1379-1384. doi: 10.1161/01.STR.0000221815.64093.8c

\section{ACKNOWLEDGMENTS}

This work was partly supported by the Scientific \& Technological Cooperation with China Project No. CN 06/2020 of the Austrian Agency for International Cooperation in Education and Research (OEAD) and the Federal Minister of Education, Science, and Research (BMBWF), Austria.

Forkel, S. J., Thiebaut de Schotten, M., Dell'Acqua, F., Kalra, L., Murphy, D. G., Williams, S. C., et al. (2014). Anatomical predictors of aphasia recovery: a tractography study of bilateral perisylvian language networks. Brain. 137, 2027-2039. doi: 10.1093/brain/awu113

Fridriksson, J., Yourganov, G., Bonilha, L., Basilakos, A., Den Ouden, D. B., and Rorden, C. (2016). Revealing the dual streams of speech processing. Proc. Natl. Acad. Sci. U.S.A. 113, 15108-15113. doi: 10.1073/pnas.161403 8114

Garrod, S., and Pickering, M. J. (2016). Dual-stream accounts bridge the gap between monkey audition and human language processing: Comment on "Towards a Computational Comparative Neuroprimatology: Framing the language-ready brain” by Michael Arbib. Phys Life Rev. 16, 69-70. doi: 10.1016/ j.plrev.2016.01.008

Goucha, T., Zaccarella, E., and Friederici, A. D. (2017). A revival of Homo loquens as a builder of labeled structures: Neurocognitive considerations. Neurosci Biobehav Rev . 81, 213-224. doi: 10.1016/j.neubiorev.2017.01.036

Heiss, W. D., Thiel, A., Kessler, J., and Herholz, K. (2003). Disturbance and recovery of language function: correlates in PET activation studies. Neuroimage 20, S42-S49. doi: 10.1016/j.neuroimage.2003.09.005

Hickok, G., and Poeppel, D. (2004). Dorsal and ventral streams: a framework for understanding aspects of the functional anatomy of language. Cognition 92, 67-99. doi: 10.1016/j.cognition.2003.10.011

Hickok, G., and Poeppel, D. (2007). The cortical organization of speech processing. Nat Rev Neurosci. 8, 393-402. doi: 10.1038/nrn 2113

Hillis, A. E., Kleinman, J. T., Newhart, M., Heidler-Gary, J., Gottesman, R., Barker, R. P., et al. (2006). Restoring cerebral blood flow reveals neural regions critical for naming,". The Journal of Neuroscience 26, 8069-8073.

Ivanova, M. V., Isaev, D. Y., Dragoy, O. V., Akinina, Y. S., Petrushevskiy, A. G., Fedina, O. N., et al. (2016). Diffusion-tensor imaging of major white matter tracts and their role in language processing in aphasia. Cortex 85, 165-181. doi: 10.1016/j.cortex.2016.04.019

Kiran, S., Meier, E. L., Kapse, K. J., and Glynn, P. A. (2015). Changes in task-based effective connectivity in language networks following rehabilitation in poststroke patients with aphasia. Front Hum Neurosci. 9:316. doi: 10.3389/fnhum. 2015.00316

Kreisler, A., Godefroy, O., Delmaire, C., Debachy, B., Leclercq, M., Pruvo, J.P., et al. (2000). The anatomy of aphasia revisited. Neurology 54, 1117-1123. doi: 10.1093/brain/awx363

Kümmerer, D., Hartwigsen, G., Kellmeyer, P., Glauche, V., Mader, I., Klöppel, S., et al. (2013). Damage to ventral and dorsal language pathways in acute aphasia. Brain 136, 619-629. doi: 10.1093/brain/aws354

Leppänen, P. H. T., Tóth, D., Honbolygó, F., Lohvansuu, K., Hämäläinen, J. A., Demonet, J. F., et al. (2019). Reproducibility of Brain Responses: High for Speech Perception, Low for Reading Difficulties. Sci Rep . 9, 8487. doi: 10.1038/ s41598-019-41992-7

Lukic, S., Barbieri, E., Wang, X., Caplan, D., Kiran, S., Rapp, B., et al. (2017). Right Hemisphere Grey Matter Volume and Language Functions in Stroke Aphasia. Neural Plast. 2017, 5601509. doi: 10.1155/2017/560 1509

Lyden, P. D., Lu, M., Levine, S. R., Brott, T. G., and Broderick, J. (2001). A modified National Institutes of Health Stroke Scale for use in stroke clinical trials: preliminary reliability and validity. Stroke 32, 1310-1317. doi: 10.1161/ 01.str.32.6.1310

Marebwa, B. K., Fridriksson, J., Yourganov, G., Feenaughty, L., Rorden, C., and Bonilha, L. (2017). Chronic post-stroke aphasia severity is determined by 
fragmentation of residual white matter networks. Sci Rep. 7, 8188. doi: 10.1038/ s41598-017-07607-9

McKinnon, E. T., Fridriksson, J., Basilakos, A., Hickok, G., Hillis, A. E., Spampinato, M. V., et al. (2018). Types of naming errors in chronic poststroke aphasia are dissociated by dual stream axonal loss. Sci Rep. 8, 14352. doi: 10.1038/s41598-018-32457-4

Musso, M., Weiller, C., Kiebel, S., Müller, S. P., Bülau, P., and Rijntjes, M. (1999). Training-induced brain plasticity in aphasia. Brain 122(Pt 9), 1781-1790. doi: 10.1093/brain/122.9.1781

Naeser, M. A., Martin, P. I., Baker, E. H., Hodge, S. M., Sczerzenie, S. E., Nicholas, M., et al. (2004). Overt propositional speech in chronic nonfluent aphasia studied with the dynamic susceptibility contrast fMRI method. Neuroimage 22, 29-41. doi: 10.1016/j.neuroimage.2003.11.016

Northam, G. B., Adler, S., Eschmann, K. C. J., Chong, W. K., Cowan, F. M., and Baldeweg, T. (2018). Developmental conduction aphasia after neonatal stroke. Ann. Neurol. 83, 664-675. doi: 10.1002/ana.25218

Nouwens, F., Visch-Brink, E. G., El Hachioui, H., Lingsma, H. F., van de SandtKoenderman, M. W. M. E., Dippel, D. W. J., et al. (2018). Validation of a prediction model for long-term outcome of aphasia after stroke. BMC Neurol. 18:170. doi: 10.1186/s12883-018-1174-5

Pani, E., Zheng, X., Wang, J., Norton, A., and Schlaug, G. (2016). Right hemisphere structures predict poststroke speech fluency. Neurology. 86, 1574-1581. doi: $10.1212 /$ wnl.0000000000002613

Rosso, C., Vargas, P., Valabregue, R., Arbizu, C., Henry-Amar, F., Leger, A., et al. (2015). Aphasia severity in chronic stroke patients: a combined disconnection in the dorsal and ventral language pathways. Neurorehabilitation and Neural Repair 29, 287-295. doi: 10.1177/1545968314543926

Rueckert, D., Sonoda, L. I., Hayes, C., Hill, D. L., Leach, M. O., and Hawkes, D. J. (1999). Nonrigid registration using free-form deformations: application to breast MR images. IEEE Trans Med Imaging 18, 712-721. doi: 10.1109/42. 796284

Sammler, D., Grosbras, M. H., Anwander, A., Bestelmeyer, P. E., and Belin, P. (2015). Dorsal and Ventral Pathways for Prosody. Curr. Biol. 25, 3079-3085. doi: 10.1016/j.cub.2015.10.009

Saur, D., Kreher, B. W., Schnell, S., Kümmerer, D., Kellmeyer, P., Vry, M. S., et al. (2008). Ventral and dorsal pathways for language. Proc. Natl. Acad. Sci. U.S.A. 105, 18035-18040. doi: 10.1073/pnas.0805234105

Saur, D., Lange, R., Baumgaertner, A., Schraknepper, V., Willmes, K., Rijntjes, M., et al. (2006). Dynamics of language reorganization after stroke. Brain 129, 1371-1384. doi: 10.1093/brain/aw1090

Smith, S. M. (2002). Fast robust automated brain extraction. Hum Brain Mapp 17, 143-155. doi: 10.1002/hbm.10062

Smith, S. M., Jenkinson, M., Johansen-Berg, H., Rueckert, D., Nichols, T. E., Mackay, C. E., et al. (2006). Tract-based spatial statistics: voxelwise analysis of multi-subject diffusion data. Neuroimage 31, 1487-1505. doi: 10.1016/j. neuroimage.2006.02.024

Smith, S. M., Jenkinson, M., Woolrich, M. W., Beckmann, C. F., Behrens, T. E., Johansen-Berg, H., et al. (2004). Advances in functional and structural MR image analysis and implementation as FSL. Neuroimage 23, S208-S219. doi: 10.1016/j.neuroimage.2004.07.051

Smith, S. M., and Nichols, T. E. (2009). Threshold-free cluster enhancement: addressing problems of smoothing, threshold dependence and localisation in cluster inference. Neuroimage 44, 83-98. doi: 10.1016/j.neuroimage.2008.03. 061

Tan, L. H., Laird, A. R., Li, K., and Fox, P. T. (2005). Neuroanatomical correlates of phonological processing of Chinese characters and alphabetic words: a meta-analysis. Hum Brain Mapp. 25, 83-91. doi: 10.1002/hbm.20134

Tao, J., Fang, Y., Wu, Z., Rao, T., Su, Y., Lin, L., et al. (2014). Community-applied research of a traditional Chinese medicine rehabilitation scheme on Broca's aphasia after stroke: study protocol for a randomized controlled trial. Trials. 15, 290. doi: 10.1186/1745-6215-15-290

Thiel, A., Schumacher, B., Wienhard, K., Gairing, S., Kracht, L. W., Wagner, R., et al. (2006). Direct demonstration of transcallosal disinhibition in language networks. J. Cereb. Blood Flow Metab. 26, 1122-1127. doi: 10.1038/sj.jcbfm. 9600350
Turkeltaub, P. E., Coslett, H. B., Thomas, A. L., Faseyitan, O., Benson, J., Norise, C., et al. (2012). The right hemisphere is not unitary in its role in aphasia recovery. Cortex. 48, 1179-1186. doi: 10.1016/j.cortex.2011.06.010

Valaki, C. E., Maestu, F., Simos, P. G., Zhang, W., Fernandez, A., Amo, C. M., et al. (2004). Cortical organization for receptive language functions in Chinese, English, and Spanish: a cross-linguistic MEG study. Neuropsychologia 42, 967979. doi: 10.1016/j.neuropsychologia.2003.11.019

Veale, J. F. (2014). Edinburgh Handedness Inventory - Short Form: a revised version based on confirmatory factor analysis. Laterality. 19, 164-177. doi: 10.1080/1357650X.2013.783045

Weiller, C., Isensee, C., Rijntjes, M., Huber, W., Müller, S., Bier, D., et al. (1995). Recovery from Wernicke's aphasia: a positron emission tomographic study. Ann. Neurol. 37, 723-732. doi: 10.1002/ana.410370605

Wilke, M., and Lidzba, K. (2007). LI-tool: A new toolbox to assess lateralization in functional MR-data. Journal of Neuroscience Methods. 163, 128-136.

Winhuisen, L., Thiel, A., Schumacher, B., Kessler, J., Rudolf, J., Haupt, W. F., et al. (2005). Role of the contralateral inferior frontal gyrus in recovery of language function in poststroke aphasia: a combined repetitive transcranial magnetic stimulation and positron emission tomography study. Stroke . 36, 1759-1763. doi: 10.1161/01.STR.0000174487.81126.ef

Wong, P. C., Warrier, C. M., Penhune, V. B., Roy, A. K., Sadehh, A., Parrish, T. B., et al. (2007). Volume of Left Heschl's Gyrus and Linguistic Pitch Learning. Cerebral Cortex 18, 828-836. doi: 10.1093/cercor/bhm115

Xing, S., Lacey, E. H., Skipper-Kallal, L. M., Jiang, X., Harris-Love, M. L., Zeng, J., et al. (2016). Right hemisphere grey matter structure and language outcomes in chronic left hemisphere stroke. Brain. 139, 227-241. doi: 10.1093/brain/awv323

Yang, X. Y., Wang, L. Q., Li, J. G., Liang, N., Wang, Y., and Liu, J. P. (2018). Chinese herbal medicine Dengzhan Shengmai capsule as adjunctive treatment for ischemic stroke: A systematic review and meta-analysis of randomized clinical trials. Complement Ther Med. 36, 82-89. doi: 10.1016/j.ctim.2017.12. 004

Zhang, Q. S., Ji, S. R., Li, S. L., He, Y., Jia, G. H., Qin, J. T., et al. (2005). Reliability and validity of Chinese rehabilitation research center standard aphasia examination. Chin J Rehabil Theory Practice 11, 703-705.

Zhao, J., Guo, J., Zhou, F., and Shu, H. (2011). Time course of Chinese monosyllabic spoken word recognition: evidence from ERP analyses. Neuropsychologia. 49, 1761-1770. doi: 10.1016/j.neuropsychologia.2011.02.054

Zhu, D., Chang, J., Freeman, S., Tan, Z., Xiao, J., Gao, Y., et al. (2014). Changes of functional connectivity in the left frontoparietal network following aphasic stroke. Front Behav Neurosci. 8:167. doi: 10.3389/fnbeh.2014.00167

Zhu, L., Nie, Y., Chang, C., Gao, J. H., and Niu, Z. (2014). Different patterns and development characteristics of processing written logographic characters and alphabetic words: an ALE meta-analysis. Hum Brain Mapp . 35, 2607-2618. doi: 10.1002/hbm. 22354

Zündorf, I. C., Lewald, J., and Karnath, H. O. (2016). Testing the dual-pathway model for auditory processing in human cortex. Neuroimage 124, 672-681. doi: 10.1016/j.neuroimage.2015.09.026

Conflict of Interest: The authors declare that the research was conducted in the absence of any commercial or financial relationships that could be construed as a potential conflict of interest.

Publisher's Note: All claims expressed in this article are solely those of the authors and do not necessarily represent those of their affiliated organizations, or those of the publisher, the editors and the reviewers. Any product that may be evaluated in this article, or claim that may be made by its manufacturer, is not guaranteed or endorsed by the publisher.

Copyright $(2021$ Fan, Gao, Zhang, Xin, Sang, Tan, Zhang, Li, Huang, Li and Chang. This is an open-access article distributed under the terms of the Creative Commons Attribution License (CC BY). The use, distribution or reproduction in other forums is permitted, provided the original author(s) and the copyright owner(s) are credited and that the original publication in this journal is cited, in accordance with accepted academic practice. No use, distribution or reproduction is permitted which does not comply with these terms. 